Jpn. J. Med. Sci. Biol., 45, 215 - 230, 1992.

\title{
SEROSURVEILLANCE FOR PSEUDOMONAS PSEUDOMALLEI INFECTION IN THAILAND
}

\author{
Pimjai NAIGOWTT, Wanchai MANEEBOONYOUNG, \\ PiyadaWONGROONSUB, Vipada CHAOWAGUL1 1 and Koomi KANAI*
}

National Institute of Health, Department of Medical Sciences, 88/7 Soi Bamrasnaradura Hospital, Tivanon Road, Nonthaburi 11000, Thailand and 1Sappasitthiprasong Ubon Hospital, Ubon Ratchathani, Thailand

(Received October 12, 1992. Accepted February 1, 1993)

SUMMARY: A nation-wide survey was conducted to see the prevalence of serosensitivity to Pseudomonas pseudomallei antigens by indirect hemagglutination (IHA) and indirect immunofluorescent assay (IFA) for IgG and IgM. Serum samples were collected from blood donors in eight selected areas and bacteriologically confirmed melioidosis patients in Ubon Ratchathani province. The distribution patterns of antibody titers were compared among the survey areas with cut-off points set at 1:160 for IHA, 1:4 for IFA-IgM and 1:32 for IFAIgG. These cut-off points were decided by ROC (Receiver Operating Characteristics) analysis. The specificity (\% true negative reactions) of each serological test in the general population differed significantly among survey areas, possibly reflecting the extent of inapparent infection in each community. IFA was more successful than IHA in differentiating between negative from positive reactions. The survey classified the areas into endemic (Khon Kaen, Ubon Ratchathani), transported (Bangkok), and non-endemic (other provinces) types.

*Reprint requests to Dr. Koomi Kanai, National Institute of Health, Department of Medical Sciences, 88/7 Soi Bamrasnaradura Hospital, Tivanon Road, Nonthaburi 11000, Thailand. 


\section{INTRODUCTION}

Melioidosis is caused by infection with $P$. pseudomallei. The disease spectrum is so wide from subclinical to septicemic with a variety of clinical symptoms, resembling other acute and chronic bacterial infections. Thus, melioidosis is often called "The great imitator" (1). Definitive diagnosis must, therefore, be made only by positive culture of $P$. pseudomallei, not by positive serology.

Nevertheless, great efforts have been made by several authors to devise or improve serological assay methods for the diagnosis of melioidosis (2-5), since serological assay can be more routinely performed in usual laboratories providing another piece of information for diagnosis, if not definitive. Especially for epidemiological survey of inapparent infections or subclinical cases, serological assay is the only one procedure available for the purpose.

It has been known that, though meilioidosis is not always a common disease, inapparent infection with $P$. pseudomallei is rather prevalent in endemic areas $(6,7)$. In Thailand, however, no nation-wide serosurvey has ever been conducted to see the prevalence of serosensitivity to $P$. pseudomallei antigen in the whole country. The presence of endemic areas has been known only by the number of reported cases from the hospitals where clinical awareness of this infectious disease is particuarly high.

In the present study, a serosurvey was conducted for P. seudomallei infection with serum samples collected from blood donors in eight selected provinces. As assay methods, indirect hemagglutination (IHA), immunofluorescent assay for IgG (IFA-IgG) and immunofluorescent assay for IgM (IFAIgM) were employed to obtain the frequency distribution patterns of antibody titers in each survey group. The data were subjected to Receiver Operating Characteristics (ROC) analysis and $\chi^{2}$ test to evaluate the epidemiological significance $(8,9)$.

\section{MATERIALS AND METHODS}

Serum specimens: Serum specimens were collected from healthy blood donors at eight selected blood centers located in Lampang, Phisanulok, Khon Kaen, Ubon Ratchathani, Bangkok, Chonburi, Suratthani, and Songkhla (Fig. 1). 


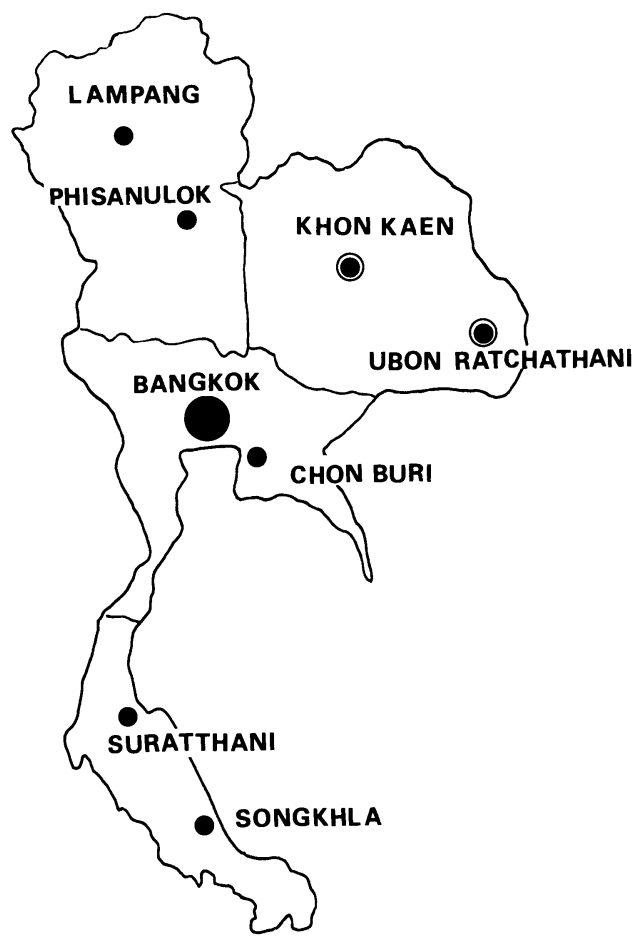

Fig. 1. Location of survey targets selected as melioidosis-endemic ( $O$ ) and non-endemic areas $(\boldsymbol{O})$.

For the positive control sera, those from melioidosis patients were obtained from Sappasitthiprasong Ubon Hospital. On the other hand, another set of 50 sera were collected in the same hospital from septicemic cases from which bacterial species other than $P$. pseudomallei were isolated as causative organisms. These species were other Pseudomonas species, staphylococci, E. coli, Klebsilla, and Salmonella.

IHA test: The IHA test was performed in a microtiter system with $P$. pseudomallei culture filtrate as antigen. The antigen was prepared from bacterial culture in glycine broth incubated at $37 \mathrm{C}$ for 14 days. The culture was killed at $121 \mathrm{C}$ for $15 \mathrm{~min}$ and centrifuged at 4,000 rpm for $1 \mathrm{hr}$. The supernatant was used as antigen. Saline-washed sheep erythrocytes were sensitized by incubating them with the antigen at $37 \mathrm{C}$ for $1 \mathrm{hr}$, and the excess antigen was removed by washing three times with isotonic saline. Serm specimens were inactivated at $56 \mathrm{C}$ for $30 \mathrm{~min}$ and absorbed with saline-washed nonsensitized sheep erythrocytes $(5 \%)$ at room temperature for $30 \mathrm{~min}$ before testing. Serial twofold dilutions $(1: 10$ to $1: 10,240)$ were made from thus inactivated serum 
specimens in 0.15 M PBS, and each dilution was incubated with the sensitized erythrocytes for $2 \mathrm{hr}$ at room temperature. The endpoint titer was defined as the highest dilution in which hemagglutination occurred.

IFA test: IFA was conducted for IgG and IgM antibodies to $P$. pseumomallei. A PBS suspension of $P$. pseudomallei of $10^{9}$ cells per $\mathrm{ml}$ was prepared from an overnight growth of a local strain from our collection. One drop thereof was placed on a slide glass and air-dried to be followed by fixation with acetone for 15 min. The fixed specimens were exposed to serial twofold dilutions in PBS of test serum samples and incubated in a moist chamber at $37 \mathrm{C}$ for $45 \mathrm{~min}$. The smears were then washed three times with fresh PBS, and air-dried. Finally, the smears were treated with $20 \mu$ l of a 1:20 dilution of fluorescein isothiocyanate-human IgG or -human IgM conjugate and incubated in a moist chamber at $37 \mathrm{C}$ for $45 \mathrm{~min}$. After washing and drying, the smears were subjected to fluorescent microscopy.

Mathematiacal treatment: The specificity, sensitivity and accuracy of IHA, IFA-IgG and IFA-IgM were calculated according to ROC analysis $(8,9)$ of the frequency distribution patterns of antibody titers in the groups of blood donors and of melioidosis patients: for sensitivity, $a /(a+c) \times 100$; for specificity, $d /(b+d) \times 100$; for accuracy, $(a+d) /(a+b+c+d) \times 100$, $a$ is the number of true positive samples, $b$ is the number of false positive samples, $c$ is the number of false negative samples, and $d$ is the number of true negative samples. For the comparative evaluation of the three serological tests here employed, receiver operator characteristic curves were drawn by plotting the true positive rate against the false positive rate. The overall accuracy of a test can be evaluated as the area under the ROC curve; the larger the area, the better the test $(8,9)$.

The statistical significance for the geographical difference of the specificity (\% true negative) in IHA was examined by $\chi^{2}$-test on the original number of negative and positive reactors.

\section{RESULTS}

\section{Survey by IHA}

One hundred serum specimens of blood donors were obtained from each of all the provincial blood centers excepttha in Ubon Ratchathani. In the latter province, 135 serum specimens of blood donors and 133 of melioidosis patients were provided from Sappasitthiprasong Ubon Hospital. The sera were tested for the antibodies to $P$. pseudomallei by IHA. The distribution patterns of antibody titers in these nine groups are shown in the upper half of Table I. 


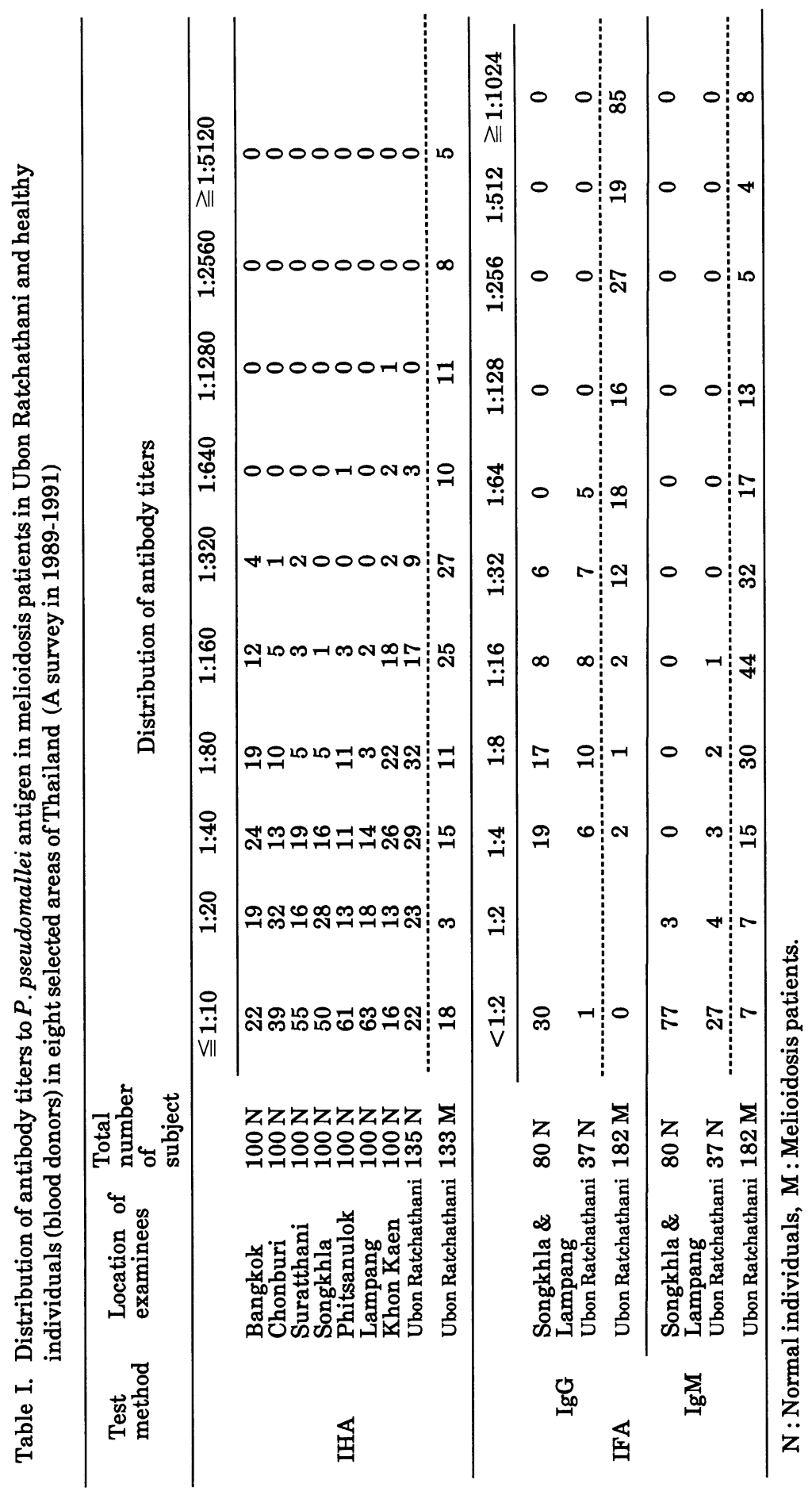


First of all, to decide the cut-off point for IHA from Table I, the following data-treatment was made by ROC analysis. According to Appassakij et al. (10) and Kunskorn et al. (11), the cut-off point was set at $\geqq 1: 160$ in their IHA test. Though this cut-off level was higher than those ever reported (12), the former authors employed this criterion in the serosurveillance for $P$. pseudomallei infection with a high specificity (92\%) and the latter authors in a patient group of melioidosis with a $98.1 \%$ specificity. If this level is adopted in the present study, 86 subjects $(10.3 \%)$ out of the total of 835 blood donors are diagnosed to have been infected with $P$. pseudomallei. In this regard, we assumed tentatively that $10 \%$ is the average prevalence rate of $P$. pseudomallei infection in Thailand.

On this general infection rate, ROC analysis $(8,9)$ was conducted to decide the cut-off levels of IHA, IFA-IgG, and IFA-IgM from the distribution patterns of antibody titers. For IHA, four levels $(1 \geqq 40,1 \geqq 80, \geqq 1: 160, \geqq 1: 320)$ were subjected to the analysis. The calculations of true positive rate (TPR) and false negative rate (FNR) were carried out with the patterns in 133 melioidosis cases in Ubon Ratchathani. False positive rate (FNR) and true negative rate (TNR) were calculated from the patterns of 500 blood donors in Suratthani, Songkhla, Phitsanulok, Lampang and Chonburi. From these four rates, "expected value" in each cut-off level was calculated and they were compared. The value in the cut-off level of $\geqq 1: 80$ and $\geqq 1: 160$ was 0.996 , being higher than 0.991 in $\geqq 1: 40$ and 0.993 in $\geqq 1: 320$. Therefore, a decision was made to use $\geqq 1: 160$ as the cut-off level of IHA.

With this criterion, the geographical difference in the specificity of IHA was surveyed in Table I and the results are summarized in Table $\Pi$. A glance at this table tells that the eight districts are divided into three groups of the different specificities as shown by dotted lines in the table. Less than $6 \%$ of the sera from Chonburi, Suratthani, Songkhla, Phitsanulok, and Lampang showed titers higehr than 1:160, while 20 to $30 \%$ of the sera from Khon Kaen and Ubon Ratchathani showed titers higher than that. The sera from Bongkok showed an intermediate pattern of the above two cases. In melioidosis patients, $65 \%$ of the total examinees the titers higher than 1:160. The significance of the differences among them was examined by $\chi^{2}$-test dealing with the actual numbers of negatives, positives, and total tests. This statistical treatment proved that the difference between Khon Kaen-Ubon Ratchathani and five provinces (Chonburi to Lampang) is significant at a probability of 0.001 , and the difference between Bangkok and the five provinces is also significant at the same probability. We tentatively assume that Khon-Kaen and Ubon Ratchathani are endemic areas, 
Table II. Geographical difference in the specificity of IHA

(Serum samples were obtained from blood donors in each province)

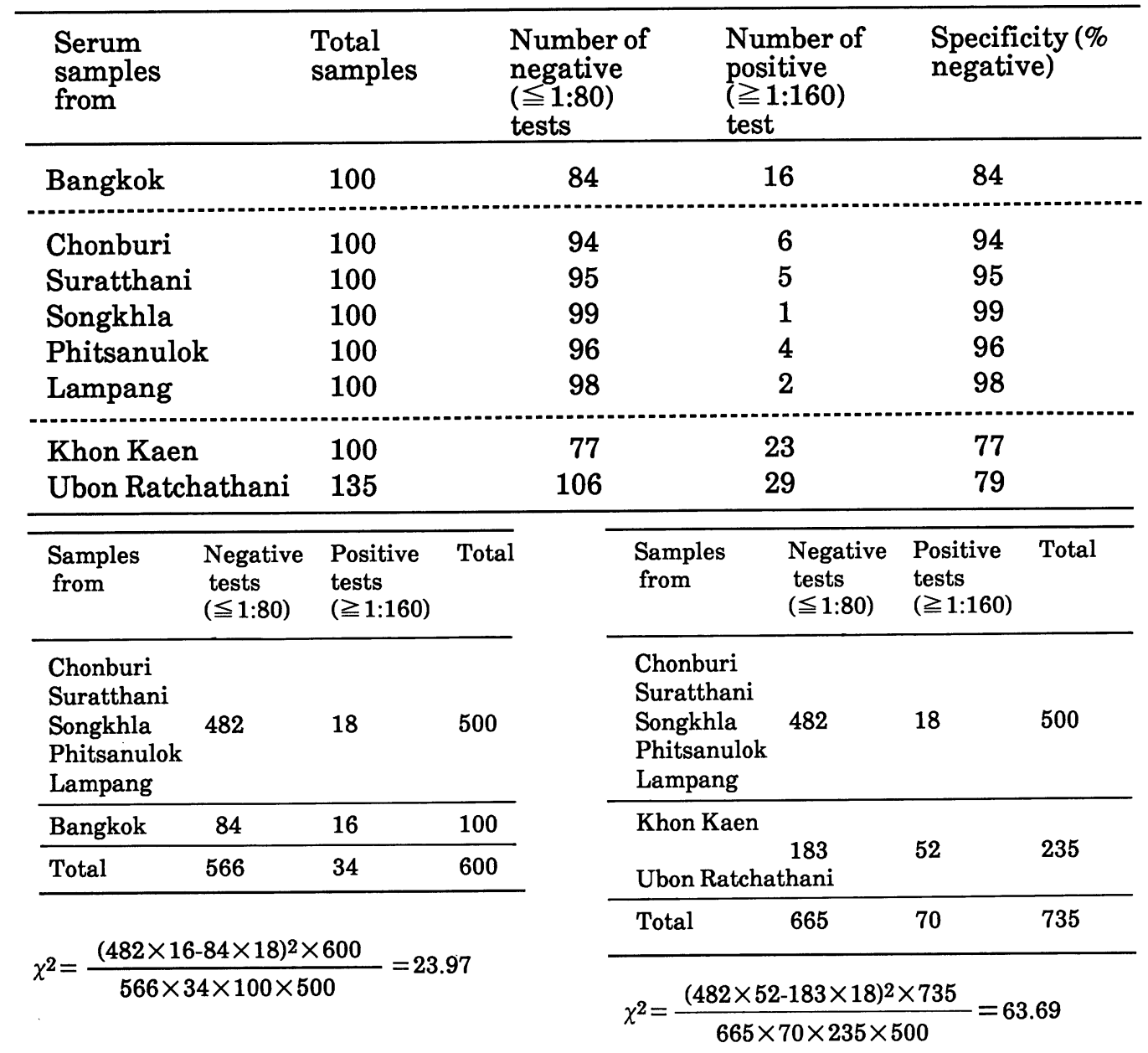


and Chonburi, Suratthani, Songkhla, Phitsanulok and Lampang are nonendemic areas.

\section{Supplemental Survey by IFA}

A supplemental serosurvey was then conducted by IFA-IgG and IFA-IgM, on 80 samples selected at random from those collected newly from blood donors in Songkhla and Lampang. Thirty-seven sera of blood donors and 182 sera of melioidosis patients were also obtained from those in Ubon Ratchathani and served for the test.

The distribution patterns of antibody titers in these tests are shown in the lower half of Table I. In order to decide the cut-off point for IFA-IgG, three levels ( $\geqq 1: 16, \geqq 1: 32,1 \geqq 1: 64$ ) were subjected to ROC analysis. TPR and FNR were calcualted from the results of 182 melioidosis patients in Ubon Ratchathani and of 80 blood donors in Songkhla and Lampang. From these rates, "expected value" in $\geqq 1: 32$ was 0.9994 , being higher than 0.9978 in $\geqq 1: 16$ and 0.9991 in $\geqq 1: 64$. Therefore, a decision was made to use $\geqq 1: 32$ as the cut-off level in IFA-IgG. In the same manner, the cut-off level for IFA-IgM was decided to be $\geqq 1: 4$, the value being 0.992 which was higher than 0.9984 in $\geqq 1: 8$ and 0.9968 in $\geqq 1: 16$.

In IFA-IgG, 6 (7.5\%) out of 80 sera from Songkhla and Lampang showed the titers higher than 1:32, but $12(32 \%)$ out of 37 sera from Ubon Ratchathani were higher than 1:32. These distribution rates were fairly corresponding rates (3\% and $29 \%$ ) of $\geqq 1: 160$ in IHA of the respective areas. On the other hand, $97 \%$ of 182 sera from melioidosis patients in Ubon Ratchathani showed titers higher than 1:32 in IFA-IgG, and 92.3\% of them were higher than 1:4 in IFA-IgM.

To evaluate the three serological assay methods employed and to get a better understanding of the infection prevalence, mathematical analysis was conducted to calculate the specificity, sensitivity and accuracy of each test. We have already known the geographical difference in the specificity of IHA. This observation may suggest that control sera must be obtained from non-endemic areas and the reliable test is possible only with them. To make this situation more clear, the specificity, sensitivity and accuracy of IHA, IFA-IgM and IFA-IgG were calculated with cut-off levels at $\geqq 1: 160, \geqq 1: 4$, and $\geqq 1: 32$, respectively, and compared the cases with control sera from an endemic area or from non-endemic areas. The results are shown in Tables III and IV. A comparison of these tables points out that the accuracy of the three tests is much improved with control sera from non-endemic areas. 
Table III. ROC analysis of melioidosis serology with control serum samples from endemic area (Ubon Ratchathani)

\begin{tabular}{lcccc}
\hline Assay & $\begin{array}{l}\text { Cut-off } \\
\text { titer }\end{array}$ & $\begin{array}{c}\text { Specificity } \\
(\%)\end{array}$ & $\begin{array}{c}\text { Sensitivity } \\
(\%)\end{array}$ & $\begin{array}{c}\text { Accuracy } \\
(\%)\end{array}$ \\
\hline IHA & $1: 160$ & 78.5 & 64.7 & 71.6 \\
& & $(\mathrm{n}=135)$ & $(\mathrm{n}=133)$ & $(\mathrm{n}=268)$ \\
IFA-IgM & $1: 4$ & 83.8 & 97.8 & 85.8 \\
& & $(\mathrm{n}=37)$ & $(\mathrm{n}=182)$ & $(\mathrm{n}=219)$ \\
IFA-OgG & \multirow{2}{*}{$1: 32$} & 67.6 & 97.3 & 92.2 \\
& & $(\mathrm{n}=37)$ & $(\mathrm{n}=182)$ & $(\mathrm{n}=219)$ \\
\hline
\end{tabular}

Control sera were obtained from blood donors in Ubon Ratchathani.

Table IV. ROC analysis of melioidosis serology with control serum samples from non-endemic areas

\begin{tabular}{lcccc}
\hline Assay & $\begin{array}{c}\text { Cut-off } \\
\text { titer }\end{array}$ & $\begin{array}{c}\text { Sepcificity } \\
(\%)\end{array}$ & $\begin{array}{c}\text { Sensitivity } \\
(\%)\end{array}$ & $\begin{array}{c}\text { Accuracy } \\
(\%)\end{array}$ \\
\hline IHA* $^{*}$ & $1: 160$ & $\begin{array}{c}96.4 \\
(\mathrm{n}=500)\end{array}$ & $\begin{array}{c}64.7 \\
(\mathrm{n}=133)\end{array}$ & $\begin{array}{c}89.9 \\
(\mathrm{n}=633)\end{array}$ \\
& & 100.0 & 92.3 & 94.6 \\
IFA-IgM** & $1: 4$ & $(\mathrm{n}=80)$ & $(\mathrm{n}=182)$ & $(\mathrm{n}=262)$ \\
& & 95.0 & 97.3 & 95.8 \\
IFA-IgG** & $1: 32$ & $(\mathrm{n}=80)$ & $(\mathrm{n}=182)$ & $(\mathrm{n}=262)$ \\
\hline
\end{tabular}

*Control sera were obtained from blood donors in four provinces; Suratthani, Songkhla, Phitsanulok, Lampang and Chonburi.

**Control sera were obtained from blood donors in two provinces;

Songkhla and Lampang.

Finally, to compare objectively capacities of the three tests, the differentiating negatives from positives ROC curves were drawn by plotting TPR against the FPR as shown in Fig. 2. The overall accuracy of each test can be evaluated from the area under the curve; the larger the area, the higher the accuracy $(8,9)$. As was expected, the higher efficiency was demonstrated in the 


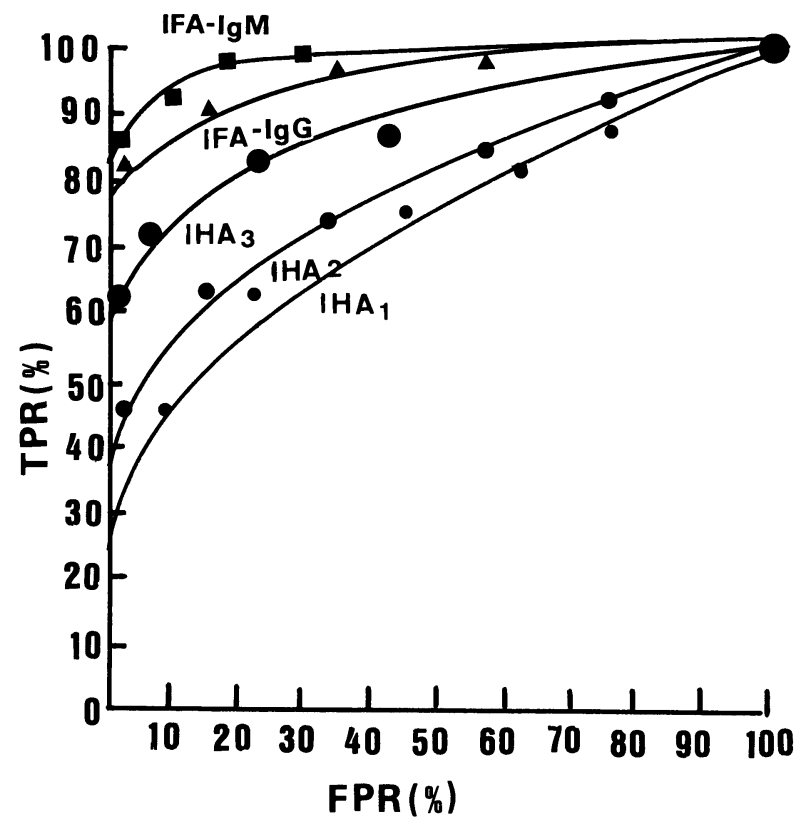

Fig. 2. ROC curves of serological assays for $P$. pseudomallei infection. (TPR: true positive rate, FPR: false positive rate). The data in Table I were the source materials for this analysis. In IHA, TPR was obtained from the patient group in Ubon Ratchathani. FPR in IHA 1 is calculated from the data of blood donors in Ubon Ratchathani, FPR in $\mathrm{IHA}_{2}$ is from those of Bangkok, and FPR in $\mathrm{IHA}_{3}$ is from those of the combined group of Suratthani, Songkhla, Phitsanulok, and Lumpang. In IFA-IgM and IFA-IgG, TPR was obtained from the data of the patient group in Ubon Ratchathani and FPR was obtained from the data of blood donors in Songkhla and Lampang.

order of IFA-IgM, IFA-IgG, and then IHA. As for IHA, a higher accuracy was obtained when the "normal" sera from the non-endemic areas were used as negative sera.

\section{Time-course Observation on the Development of Antibody Titers in Individual Patients}

In some melioidosis patients, we had the opportunities to follow the development of antibody titer at one week intervals. Figure 3 shows four selected examples as such, three from localized infection cases and one of septicemic case. The septicemic case was an acute fatal one who showed abrupt decline of titers of both IFA-IgM and IFA-IgG after they reached high levels. In three localized 


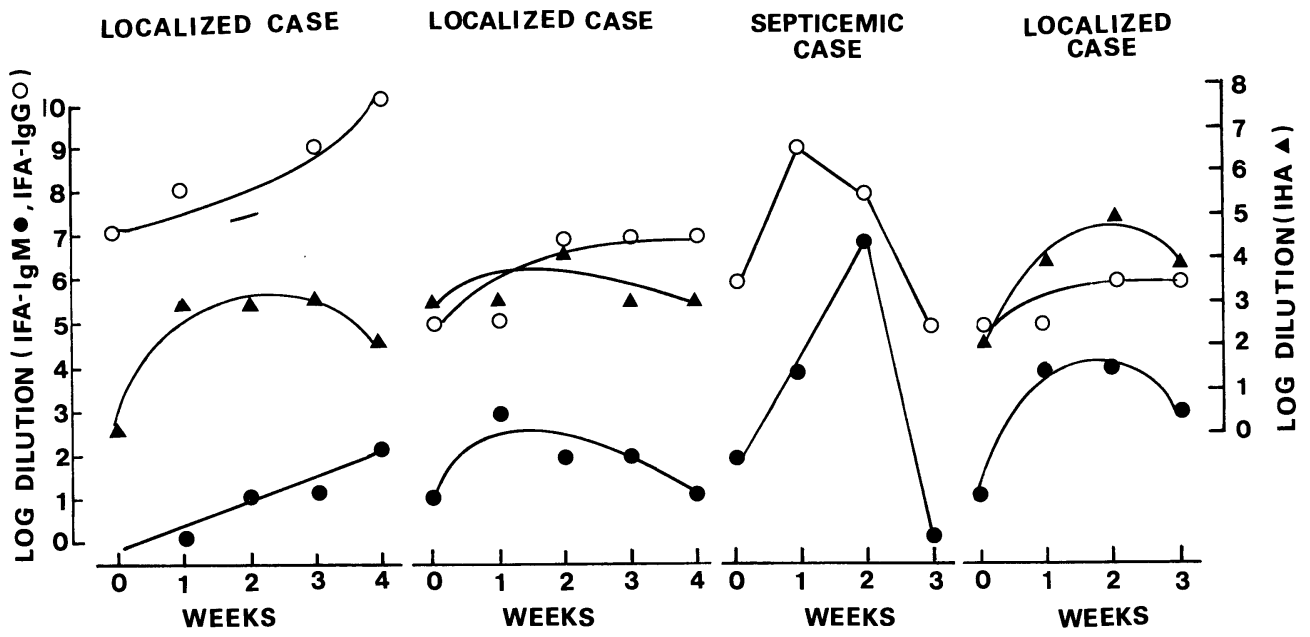

Fig.3. Examples of the development of antibody titers in melioidosis patients of localized and septicemic case.

cases, the antibody levels of IFA-IgG and IHA appeared to be more persistent than that of IFA-IgM.

\section{Serosensitivity in 50 Cases of Septicemia caused by Bacterial Species \\ Other than P. pseudomallei}

The sera of cases of septicemia due to bacterial species other than $P$. pseudomallei were tested by IHA and IFA-IgG with $P$. pseudomallei antigens. The results are shown in Table V. On the basis of our preceding experiences, we can examine this table with the antibody titers of 1:160 in IHA and 1:32 in IFAIgG as the cut-off level. Then, we have two cases of 1:160 in IHA, one is Pseudomonas spp. infection and the other is $E$. coli infection, meanwhile in IFAIgG, the occurrence rates of $\geqq 1: 32$ were more frequent, especially in Pseudomonas cepacia, staphylococci, and $E$. coli. However, there was no case having coincidently both the titers of IHA 160 and IFA-IgG 1:32. 


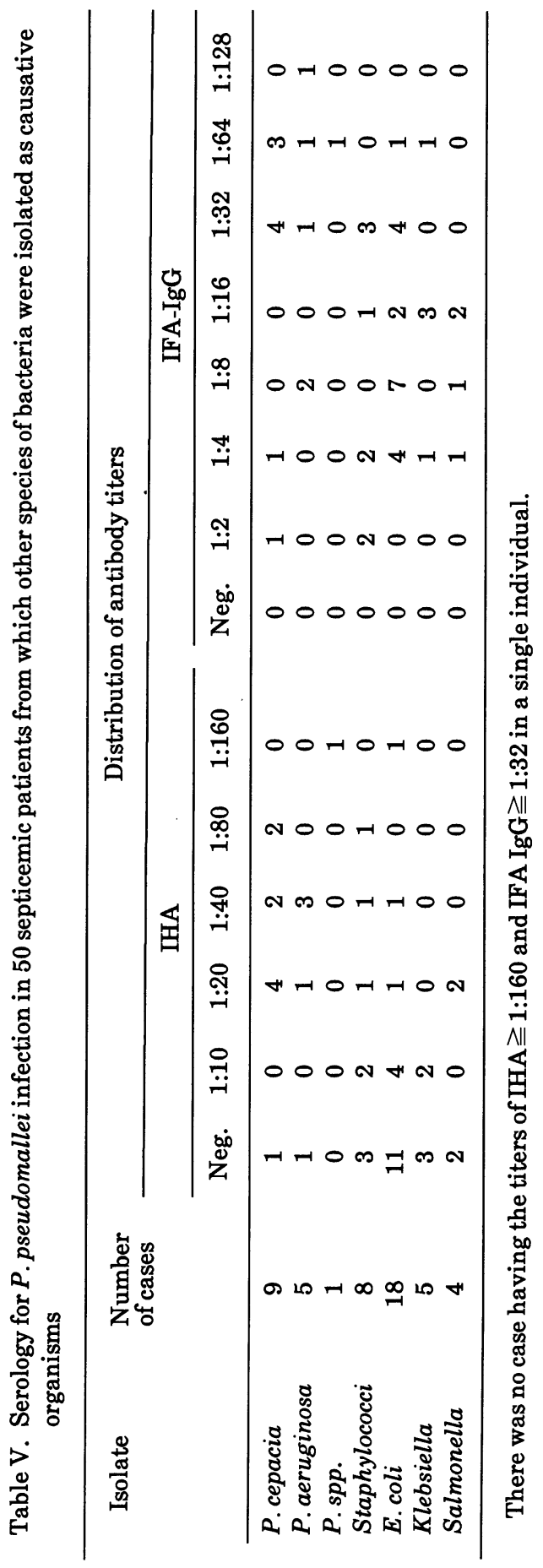




\section{DISCUSSION}

The present study aimed at a nation-wide serosurvey for $P$. pseudomallei infection and also at comparative examination of serological methods. Though there is still much discussion about the serological diagnosis of melioidosis, no other means are available for prevalence survey of inapparent infection with $P$. pseudomallei as the background epidemiology of melioidosis. The bacilli are being harbored in natural environments (soil and surface water) of endemic areas to infect human beings (12). Man-to-man transmission of melioidosis is extremely rare (13).

There may be two reasons why $P$. pseudomallei infection and melioidosis are endemic in the Northeast of Thailand. The first is the ecological situation as suggested by the field survey by Finkelstein et al. (7) that $P$. pseudomallei would be more prevalent in the natural environment of the Northeast. The second is the socio-economical reason. The inhabitants there are mostly poor farmers working in rice field. Rice field is the place of dense contamination with $P$. pseudomallei organisms. The unbalanced diet and malnutrition could be another factor for infection since melioidosis is essentially an opportunistic infection $(11,12)$. Finkelstein et al. (7) found that there was no direct correlation between the environmental density of the organisms and the local prevalence of $P$. pseudomallei infection as estimated by IHA.

In our present experiences, the occasional occurrences of cross or doubtful reactions in IHA and IFA were acknowledged in the sera of septicemic cases with the bacterial species other than P. pseudomallei. All these sera were from Ubon Ratchathani. Therefore, some of the positive reactions may have been due to inapparent infections with $P$. pseudomallei, but some others would be "true" cross reaction. If so, improvement of serological assay methods for $P$. pseudomallei infection is needed, e.g. use of purified antigen, use of monoclonal antibodies, and so on. Preparation of a diagnosis kit would be also helpful to bring such serological assay into routine application in clinical laboratories in endemic areas.

Recently, introduction of enzyme-linked immunosorbent assay (ELISA) has been attempted by Ashdown et al. (13) and Kunakorn et al (14). According to the former authors (13), the sensitivity and specificity of ELISA-IgG were evlauated at $90 \%$ and $99 \%$, respectively, comparable to those of IFA and higher than $74 \%$ sensitivity of IHA. Almost the same results were obtained in ELISA-IgM conducted by Kunakorn et al. (14), especially they recommended the combined 
use of ELISA-IgM and IHA for the serodiagnosis of melioidosis. Their method developed into gold blot for detection of IgM and IgG antibodies as an improved rapid serodiagnosis of melioidosis (15).

On the basis of the frequency of reported melioidosis cases, some provinces have been regarded as "endemic areas", e.g., Khon Kaen and Ubon Ratchathani (1). However, the frequency sometimes depends on the clinical awareness of physicians, not reflecting the actual epidemiological status. No systematic study has ever been conducted to survey for the nation-wide prevalence of $P$. pseudomallei infection. The present study was the first attempt to do this with advantage of the serum samples from healthy blood donors of eight selected provinces from the North to the South.

The overall results demonstrated that IHA and IFA in the conventional procedure and criteria were satisfactory to reveal the epidemiological status of $P$. pseudomallei infection, especially to demonstrate the geographical differences among the provinces. ROC analysis was effective in showing such differences, classifying the provinces into three epidemiological categories; endemic, nonendemic and transported areas.

Khon Kaen and Ubon Ratchathani were confirmed to be endemic areas, and the lower serological specificity of these areas was due to the more frequent presence of inapparent infections. These provinces have been providing labors to Bangkok. This may be the reason why the serological specificity in Bangkok was intermediate between endemic and other non-endemic five provinces.

As for Songkhla province, the specificity of IHA with the cut-off point at 1:160 was $99 \%$ as a good evidence of a non-endemic area. However, Appassakij et al. (10) recently reported that Songkhla was an endemic area according to their survey by IHA in the same procedure and criterion as ours, though the sensitivity being $92 \%$. We should be aware of the future epidemiological situation in Songkhla.

As for purified antigens for diagnostic serology, "exo-toxins" $(16,18)$, polysaccharide (6), and cell-surface enzymes (19) have ever been considered at the experimental level, and they are waiting for the evaluation by field study. 


\section{ACKNOWLEDGEMENTS}

Appreciation is expressed to Japan International Cooperation Agency for financial assistance to this research. We thank also Mrs. Suwanna Navacharoen for her expert assistance in preparation of the manuscript, and Dr. Saburo Iwasa for his advice in statistical analysis.

\section{REFERENCES}

1. Leelarasamee, A. and Punyagupta, S. (1989): Melioidosis. Epidemiology with Thai experience. p.238-264. In S. Punyagupta, T. Sirisanthara and B. Stapatayavong (eds.), Melioidosis, Bangkok Medical Publisher, Bangkok.

2. Alexander, A. D., Huxsoll, D. L., Warner, A. R., Jr., Shepler, V. and Dorsey, A. (1970): Serological diagnosis of human melioidosis with indirect hemagglutination and complement fixation tests. Appl. Microbiol., 20, 825833.

3. Ashdown, L. R. (1981): Demonstration of human antibodies to Pseudomonas pseudomallei by indirect fluorescent antibody staining. Pathology, 13, 597-601.

4. Ashdown, L. R. (1981): Relationship and significance of specific immunoglobulin $\mathrm{M}$ antibody response in clinical and sublinical melioidosis. J. Clin. Microbiol., 14, 361-364.

5. Khupulsup, K. and Petchclai, B. (1986): Application of indirect fluorescent antibody test for IgM antibody for diagnosis of melioidosis in Thailand. Amer. J. Trop. Med. Hyg., 35, 366-369.

6. Nigg, C. (1963): Serological studies on subclinical melioidosis. J. Immunol., 91, 18-28.

7. Finkelstein, R. A., Atthasampunna, P., Chitrakorn et al. (1966): Investigation of melioidosis in Thailand. SEATO Medical Research Study on Melioidosis. Period of Report 1 April 1965-31 March 1966, 228-234.

8. Tsuji, I. and Hisamichi, S. (1990): Receiver operating characteristics (ROC) analysis and its application to the setting up of cut-off points. Media Circle, 311-319 (text in Japanese).

9. Tsuji, I. (1990): Medical decision making -- Principle of receiver operating characteristics (ROC) analysis. Rinsho Byori (Clinical Pathology), 38, 579600 (text in Japanese).

10. Appassakij, H., Silpapojakul, K. R., Wansit, R. and Pornpakul, M. (1990): Diagnostic value of the indirect hemagglutination test for melioidosis in an endemic area. Amer. J. Trop. Med. Hyg., 42, 248-253.

11. Kunakorn, M., Boonma, P., Khupulsup, K. and Petchclai, B. (1990): Enzyme-linked immunosorbent assay for immunoglobulin $\mathbf{M}$ specific antibody for the diagnosis of melioidosis. J. Clin. Microbiol., 28, 1249-1253. 
12. Kanai, K. and Dejsirilert, S. (1988): Pseudomonas pseudomallei and melioidosis, with special reference to the status in Thailand. Jpn. J. Med. Sci. Biol., 41, 123-157.

13. Kunakarn, M., Jayanetra, P. and Tanphachitra, D. (1991): Man-to-man transmission of melioidosis. Lancet, 337, 1290-1291.

14. Ashdown, L. R., Johnson, R. W., Koehler, J. M. and Cooney, C. A. (1989): Enzyme-linked immunosorbent assay for the diagnosis of clinical and subclinical melioidosis. J. Infect. Dis., 160, 253-260.

15. Kunakorn, M., Petchclai, B., Khupulsup, K. and Naigowit, P. (1991): Gold blot for detection of immunoglobulin M (IgM)- and IgG - specific antibodies for rapid serodiagnosis of melioidosis. J. Clin. Microbiol., 29, 2065-2067.

16. Ismail, G. Mohamed, R., Rohana, S., Sharofah, H. S. M. and Embi, N. (1991): Antibody to Pseudomonas pseudomallei exotoxin in sheep exposed to natural infection. Vet. Microbiol., 27, 277-282.

17. Ismail, G., Embi, M. N., Omar, O., Allen, J. C. and Smith, C. J. (1987): A comparative immunosorbent assay for detection of Pseudomonas pseudomallei exotoxin. J. Med. Microbiol., 23, 351-357.

18. Heckley, R. J. (1964): Differentiation of exotoxin and other biologically active substances in Pseudomonas pseudomallei filtrates. J. Bacteriol., 88, 1730-1736.

19. Kondo, E., Petkanchanapong, V., Naigowit, P., Kurata, T. and Kanai, K. (1991): Demonstration of acid phosphatase activity in antigenic glycoprotein fractions obtained from culture filtrate of Pseudomonas pseudomallei. Jpn. J. Med. Sci. Biol., 44, 213-224. 